\title{
Parry-Romberg syndrome in an elderly male: A rare case report
}

\author{
Daljit Singh', Chetna Singla', Suresh K. Malhotra', Arashbir Kaur² \\ ${ }^{1}$ Department of Dermatology Venereology and Leprology Government Medical College, Amritsar, Punjab, India. ${ }^{2}$ Department \\ of Medicine, Government Medical College, Patiala, Punjab, India
}

Corresponding author: Dr. Daljit Singh, E-mail: daljit919@gmail.com

\begin{abstract}
Progressive hemifacial atrophy (PHA) of the face was first described by Caleb Hillier Parry in 1825 and Moritz Heinrich Romberg in 1846, hence the name Parry Romberg Syndrome was coined. It is a neurocutaneous syndrome characterized by progressive atrophy of the skin and tissues lying underneath like subcutaneous fat, muscle and bone. It is more common in females. Various etiologies have been proposed like autoimmune, hyperactivity of nervous system, infections etc., but none can fully explain its pathogenesis. Here we report a case of Parry-Romberg syndrome in a 70-year-old male which is the oldest case to present with this syndrome to the best of our knowledge.
\end{abstract}

Key words: Progressive hemifacial atrophy; Parry Romberg syndrome; Trigeminal neuralgia; Negative ANA; Negative dsDNA

\section{INTRODUCTION}

Progressive hemifacial atrophy (PHA) of the face was first described by Caleb Hillier Parry in 1825 and Moritz Heinrich Romberg in 1846, hence the name Parry Romberg Syndrome was coined [1]. It is a neurocutaneous syndrome characterized by progressive atrophy of the skin and tissues lying underneath like subcutaneous fat, muscle and bone. This disease is characteristically limited to the dermatomal distribution of Vth cranial nerve and occasionally involves one or two divisions of the trigeminal nerve. PHA is more common in females than in males and rarely shows family history [2]. Some authors consider it to be an autoimmune disorder and a variant of scleroderma while others think it to be caused by hyperactivity of the nervous system. An infectious cause of the disease has also been proposed by some authors [3]. The affected individuals are morphologically normal at birth and the atrophy usually occurs within the first two decades of life. We report a unique case of ParryRomberg Syndrome presenting at 70 years of age with Trigeminal Neuralgia.

\section{CASE REPORT}

A 70-year-old nonsmoker, nonalcoholic, nondiabetic male presented to our tertiary academic center with the complaint of progressive deformity of the left side of the face with severe pain since 6 months. According to the patient, he had severe pain on the left side of the face for 15-20 days after which he started noticing progressive band like thickening of the skin starting from left angle of the mouth, progressing to involve left nares, left cheek, left infraorbital region and left temporal region in that order over a period of 20 days. This band gradually deepened and the patient developed a depressed ulcer on the left vertex directed longitudinally along the midline. Concomitantly, the patient developed loss of hair over and around the affected areas of the scalp, left eyebrow and mustache.

On examination, he was moderately built and nourished, well oriented to time, place and person but was in agony due to the continuous pain from the last six months. Periorbital edema on the left side was present. Mucocutaneous examination revealed a large depressed

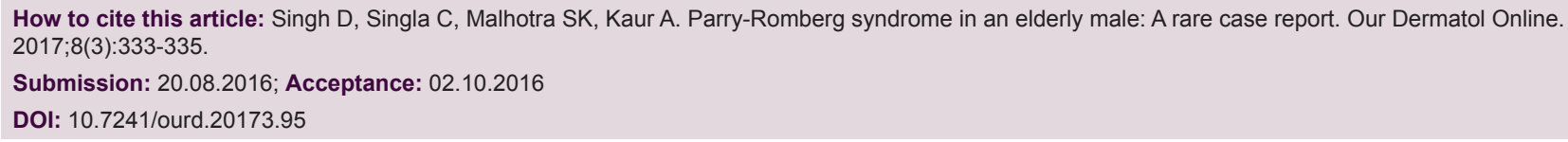


atrophic plaque limited to the ophthalmic and maxillary division of the trigeminal nerve on the left side (Fig. 1). Within the plaque, there was an ulcer arranged longitudinally along the midline on the scalp measuring $4 X 9 \mathrm{~cm}$ with sharply defined regular margins. The floor of the ulcer was clean looking (Fig. 2). A second similar small oval shaped ulcer of size $0.6 \mathrm{X} 0.5 \mathrm{~cm}$ was present on the left side of the nose. Within the plaque, there was a hypopigmented band with feathery margins extending from the left upper lip and left nares coursing along the left infraorbital and left temporal regions to meet the ulcer at its left lower margin. Few linear telangiectasias were present in the fibrotic band. This fibrotic band has led to the lifting up of the left upper lip, exposing the teeth and nibbling out of the left nasal ala. The left eye was swollen and detailed eye examination revealed vision reduced to the just perception of light. Intraoral examination revealed no significant finding as for the patient already had traumatic loss of teeth. All routine investigations including CBC, RFTs, LFTs, lipid profile, X-Ray Chest, ultrasound abdomen were within normal limits. ANA and ssDNA were not detected on serological examination. Histopathological examination of the lesional skin revealed normal looking stratified epithelium with dilated blood vessels, mild perivascular lymphocytic infiltrate and thick collagen bundles throughout the dermis (Fig. 3). Digital X-Ray of the skull in anteroposterior and lateral views revealed erosion of the parietal bone on the left side and multiple small osteolytic areas but no bony pathology was seen in the maxilla. Computed Tomography of the head and neck revealed destruction of the soft tissue as well as thinning of the parietal bone underneath the ulcer (Fig. 4). The brain was normal. Silver staining ruled out Borrelia burgdorferi infection. Based on the clinical features and investigations, the patient was diagnosed as a case of Parry-Romberg Syndrome with trigeminal neuralgia. Symptomatic treatment was given to the patient in the form of analgesics for the neuropathic pain and aseptic dressing of the ulcer was done. The pain did not improve with analgesics but showed an immediate response to Carbamazepine. The patient was planned for Dexamethasone-Methotrexate Pulse therapy and plastic surgery intervention for the ulcer but was lost to follow-up owing to his family problems and financial constraints.

\section{DISCUSSION}

Parry-Romberg Syndrome is a rare entity presenting as progressive hemifacial atrophy of the face usually



Figure 1: Atrophic plaque with feathery margins ending into an ulcer on the left side of face and scalp.

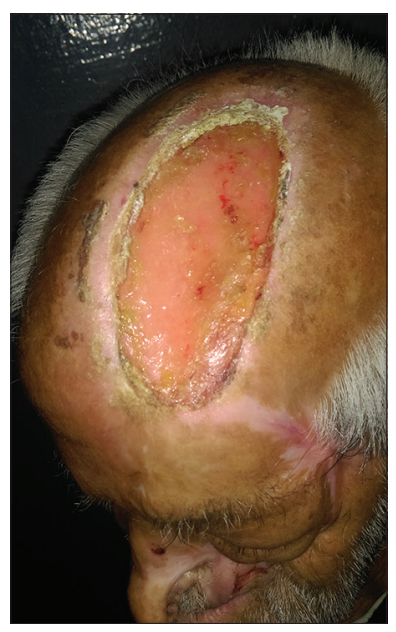

Figure 2: The ulcer strictly limited to the left side of the scalp.

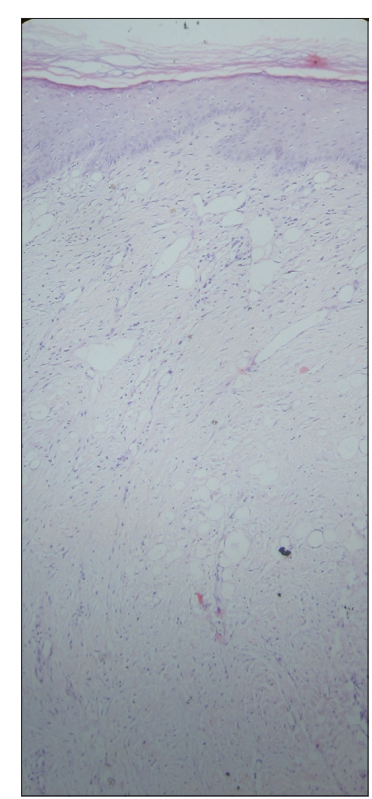

Figure 3: Normal epidermis, perivascular lymphocytic infiltrate and thick collagen bundles in the dermis. (H\&E 10X). 


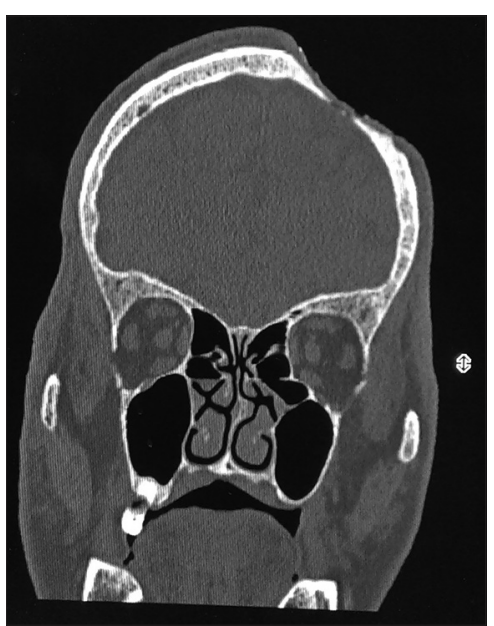

Figure 4: CT image showing resorption of soft tissue and underlying left parietal bone.

manifesting within the first decade of life [4]. It is more common in females. It can closely mimic en coup de saber morphea which can present in a similar manner, is also more common in females but usually manifests in the second to third decade of life. More strict definition of the syndrome includes contralateral jacksonian epilepsy, trigeminal neuralgia, and changes in eyes and hair [5]. Other features can be in the form of eye involvement in 10-35\% of the cases [6] presenting as enophthalmos, uveitis, ciliary body hypotrophy and phacodonesis [4]. Besides trigeminal neuralgia, there may be other neurological complications like facial paresthesias, severe headache [7]. In our case, the patient presented at 70 years of age. The clinical features and histopathology were consistent with some fibrosing disorder of the skin and underlying tissues making morphea and PHA as likely differentials.
The absence of ssDNA and ANA on serology ruled out morphea. Hence a diagnosis of Parry-Romberg Syndrome was made. Although theory supports the early manifestation of this syndrome (within the first decade) but various cases have been reported with late onset.

The case is being reported because of its rarity and late onset at 70 years of age which hasn't been reported earlier to the best of our knowledge.

\section{REFERENCES}

1. Pandey RP, Pandey A. Parry-Romberg Syndrome: A Case Report. Sch J Med Case Rep. 2014;2:214-6.

2. Blyta Y, Daka A. Localised Scleroderma- Patchy Type Morphea. J Clin Exp Dermatol Res. 2013;4:2.

3. Madasami R, Jayanandan M, Adhavan UR, Gopalakrishnan S, Mahendra L. Parry Romberg syndrome: a case report and discussion. J Oral Maxillofac Pathol. 2012;16:406-10.

4. Fea AM, Aragno V, Briamonte C, Franzone M, Putignano D, Grignolo FM. Parry Romberg syndrome with a wide range of ocular manifestations: a case report. BMC Ophthalmology. 2015;15:119.

5. Regezi JA, Scuibba JJ, Jordan RC. Oral pathology. Clinical pathological correlations. $4^{\text {th }}$ ed. USA: Saunders: Elsevier Science; 2003.

6. Whyman RA, Doyle TCA, Harding WJ, Ferguson MM. An unusual case of hemifacial atrophy. Oral Surg Oral Med Oral Pathol. 1992;73:564-69.

7. Kumar AA, Kumar RA, Shantha GPS, Aloogopinathan G. Progressive hemifacial atrophy- Parry Romberg Syndrome presenting as severe facial pain in a young man: a case report. Cases J. 2009;2:6776.

Copyright by Daljit Singh, et al. This is an open-access article distributed under the terms of the Creative Commons Attribution License, which permits unrestricted use, distribution, and reproduction in any medium, provided the original author and source are credited.

Source of Support: Nil, Conflict of Interest: None declared. 